Revue d'histoire de l'Amérique française

DEVVUE D.HISTOIRE DE L'AMÉRIQUE FRANÇAISE

\title{
L'intelligentsia du Québec et la migration des Canadiens français vers l'Ouest canadien, 1870-1930
}

\section{A.-N. Lalonde}

Volume 33, numéro 2, septembre 1979

URI : https://id.erudit.org/iderudit/303773ar

DOI : https://doi.org/10.7202/303773ar

Aller au sommaire du numéro

Éditeur(s)

Institut d'histoire de l'Amérique française

ISSN

0035-2357 (imprimé)

1492-1383 (numérique)

Découvrir la revue

Citer cet article

Lalonde, A.-N. (1979). L'intelligentsia du Québec et la migration des Canadiens français vers l'Ouest canadien, 1870-1930. Revue d'histoire de l'Amérique française, 33(2), 163-185. https://doi.org/10.7202/303773ar d'utilisation que vous pouvez consulter en ligne. 


\title{
L'INTELLIGENTSIA DU QUÉBEC ET LA MIGRATION DES CANADIENS FRANÇAIS VERS L'OUEST CANADIEN 1870-1930
}

\author{
A.-N. LALONDE \\ University of Regina
}

Les prairies canadiennes paraissaient propices à la colonisation par les Canadiens français, qui considéraient l'Ouest comme faisant partie du patrimoine hérité de leurs ancêtres. La loi créant le Manitoba garantissait aux francophones leurs droits linguistiques, ce qui avait amené George-Étienne Cartier à prédire que cette province deviendrait une réplique du Québec. La quantité des terres arables qui y étaient disponibles donna au clergé catholique l'occasion de consolider le mythe agriculturiste en détournant des États-Unis l'excédent de la population du Québec pour le diriger vers l'Ouest canadien. De 1870 à 1890 , ceux qui étaient absolument obligés de quitter le Québec furent pressés par leurs chefs de rester au Canada et de s'installer dans les prairies. Toutefois, la question des Écoles du Manitoba et la possibilité d'autres solutions, après 1890 , incitèrent les dirigeants de la société canadienne-française à modifier leurs préférences et à reléguer l'Ouest canadien à l'arrièreplan de leurs préoccupations.

L'acquisition de la Terre de Rupert, jusque-là propriété de la Hudson Bay Company, représentait pour les gouvernements d'Ottawa et de l'Ontario l'un des principaux moyens de réduire l'exode massif des Canadiens de naissance vers les Etats-Unis. L'ouverture de cette nouvelle "frontière" agricole mettrait à la disposition de la population excédentaire de l'Ontario des terres fertiles et inaugurerait une nouvelle ère de prospérité économiquel, marquée par la création de nouveaux emplois pour ceux qui, dans le passé, avaient été forcés de chercher du travail aux États-Unis.

I V. C. Fowke, The National Policy and the Wheat Economy (University of Toronto, 1957), 10-16.

$$
\text { [163] }
$$

RHAF, vol. 33, no 2, septembre 1979 
Pendant ce temps, les Canadiens français ne voyaient, dans l'achat de cette "terre infertile", de cette "solitude désertique", de ce "gouffre à engloutir nos taxes"2, qu'un gaspillage des fonds publics. Alors que le ministère Macdonald était à préparer la loi qui devait pourvoir à la construction du chemin de fer du Pacifique canadien, de manière à faciliter la colonisation dans les prairies, les hommes politiques du Québec discutaient des voies et moyens pour la construction de routes de colonisation et de chemins de fer, afin de donner à l'excédent de population de la province accès à de nouvelles terres fertiles ${ }^{3}$. Au cours des années 1870 et 1880 , une multitude de sociétés de colonisation et de corporations furent créées partout en Ontario, par des hommes d'affaires, des hommes d'Église et des hommes politiques, dans le but avoué de détourner vers le Manitoba et le Nord-Ouest le courant migratoire qui portait les jeunes Canadiens vers les États-Unis ${ }^{4}$. Au Québec, environ cinquante sociétés de colonisation aidaient de diverses manières les citoyens sans ressources à se procurer un nouvel établissement à l'intérieur de la provinces.

C'est le clergé de l'Ouest canadien qui, sous la direction de Mgr Alexandre Taché, archevêque de Saint-Boniface, incita le clergé et les hommes politiques du Québec à accorder quelque attention au Manitoba et au Nord-Ouest comme territoires possibles de colonisation pour les Canadiens français. À la suite de l'appel lancé personnellement par Mgr Taché, on prépara et distribua une lettre circulaire, portant la signature de chacun des évêques du Québec, qui devait être lue du haut de la chaire dans toutes les églises de la province. Dans cette lettre, datée du 23 octobre 1871, les dirigeants de l'Église catholique déploraient l'exode des Canadiens français vers les États-Unis, louaient le travail du clergé dans le domaine de la colonisation au sein de la province et recommandaient qu'on encourageât ceux qui étaient absolument obligés de quitter le Québec à rester en terre canadienne en s'établissant au Manitoba:

2 R. Rumilly, Histoire de la province de Québec (Éditions Bernard Valiquette, Montréal (s.d.), I: 158-59.

3 Marcel Hamelin, Les premières années du parlementarisme québécois, 1867. 1878 (Les Presses de l'Université Laval, Québec, 1974), 73-117.

4 A. N. Lalonde, Settlement in the North-West Territories by Colonization Companies. Thèse manuscrite (PH. D.), (Université Laval, 1969), 32-73.

5 Marcel Hamelin, op. cit., 92-94. 
L'acquisition du Nord-Ouest, la création de la province du Manitoba offrent un avantage réel à ceux qui n'aiment pas le défrichement des terres boisées et qui pourtant voudraient s'éloigner de la paroisse qu'ils habitent.

Notre pensée n'est pas de demander aux paisibles et heureux habitants de la province de Québec, de changer une position certaine et avantageuse pour les incertitudes et les risques d'une émigration lointaine, mais s'il en est auxquels il faut un changement et auxquels il répugne de s'imposer les rudes labeurs de bûcherons, à ceux-là, monsieur le curé, veuillez bien indiquer la province de Manitoba. ${ }^{6}$

En d'autres mots, la hiérarchie de l'Église catholique au Québec affirmait clairement que le premier remède à l'expatriation demeurait la colonisation de nouvelles terres à l'intérieur même de la province. Cependant, à ceux qui étaient contraints de quitter le Québec, elle conseillait d'émigrer dans l'Ouest canadien, où, par leur simple présence, ils contribueraient à maintenir un certain équilibre entre les deux races fondatrices au sein de la Chambre des Communes, à Ottawa.

L'acceptation ainsi exprimée, du Manitoba comme territoire de colonisation pour une partie de la population excédentaire du Québec, suscita, pendant les années 1870 , un esprit assez général de collaboration entre les membres du clergé et les laïcs éminents de l'Ouest et de l'Est. Le Père Lacombe fut invité à prendre place sur la même tribune que le premier ministre du Québec pour lancer un appel spécial en vue d'obtenir des colons pour le Manitoba, pendant la "Convention générale des Canadiens français" organisée par la Société Saint-Jean-Baptiste de Montréal en juin 18747. En 1876, Mgr Bourget donna sa bénédiction à la création d'une société de colonisation formée de plusieurs des principaux citoyens de Montréal, qui travaillerait de concert avec la Société de colonisation du Manitoba pour diminuer le nombre des départs vers les États-Unis et pour promouvoir la colonisation francophone dans les prairies ${ }^{8}$.

Archdiocesan Archives of Regina, Immigration and Colonization, Copie de cette lettre circulaire; elle parut dans La Presse, le 5 avril 1890, et dans Mgr A. Taché, Esquisse sur le Nord-Ouest de l'Amérique (Beauchemin, Montréal, 1901), 178-79. Montréal), 104

7 Rumilly, Histoire de la Société Saint-Jean-Baptiste de Montréal (L'Aurore,

${ }_{8}$ Le Nouveau Monde, 18 mai 1876. Alfred La Rocque et le Dr C. M. Lachapelle sont nommés parmi les membres de cette société. Voir aussi Les Cloches de Saint-Boniface, ler août 1916. 
Même le curé Labelle, de Saint-Jérôme, envoya dans la province récemment créée de l'Ouest, en avril 1879 , un contingent de quinze colons des comtés de Terrebonne, de l'Assomption et du Lac-desDeux-Montagnes 9 . La majorité des principaux journaux du Québec appuyaient les efforts du clergé de l'Ouest pour rapatrier les Canadiens français exilés aux États-Unis et ses tentatives pour diriger vers le Manitoba les francophones qui projetaient de quitter la belle province ${ }^{10}$.

Ce n'est pas tout le monde, cependant, qui, au Québec, secondait l'action du clergé de l'Ouest. Dans une lettre à son supérieur, Mgr Taché, le Père Lacombe rapportait le détail d'une discussion qu'il avait eue en 1876 avec le premier ministre du Québec, Boucher de Boucherville: "Loin de m'encourager pour notre affaire d'émigration il m'a assuré qu'il ferait tout en son pouvoir pour empêcher les Canadiens de la province de Québec d'émigrer à Manitoba. Il m'a dit d'aller chercher ceux des ÉtatsUnis et puis voilà..." 11 Certains journaux mettaient en doute les descriptions enthousiastes des prairies canadiennes faites par les membres du clergé de l'Ouest, alors que Mgr Taché lui-même, dans son Esquisse sur le Nord-Ouest de l'Amérique, avait exprimé de sérieuses réserves sur la fertilité du sol dans plusieurs régions de l'Ouest canadien et sur l'opportunité d'une migration de Canadiens français du Québec vers ces régions inhospitalières. À plusieurs reprises ces accusations contre Mgr Taché refirent surface dans la presse, au cours des années 1880 et 1890 . L'éminent archevêque tenta vainement de démontrer la fausseté de ces accusations et de se disculper dans une série de réponses bien documentées, mais les doutes et les soupçons persistèrent longtemps après sa mort ${ }^{12}$. Fondées ou non, ces accusations eurent une influence considérable, comme en témoigne cet extrait du journal de Mgr O.-E. Mathieu, alors premier évêque de Régina, qui avait été recteur de l'Université Laval avant d'être élevé à l'épiscopat en 1910:

9 F. M. Shimnowski, Douze années d'immigration française au Manitoba 18601882, Thèse manuscrite (M.A.) (Université d'Ottawa, 1950), 90.

10 R. Painchaud, The Catholic Church and the Movement of Francophones to the Canadian Prairies 1870-1915, Thèse manuscrite (Ph.D.) (Université d'Ottawa, 1976), 169-71.

11 Archives de l'Archidiocèse de Saint-Boniface, Fonds Taché, Lacombe à Taché, Montréal, ler mars 1876; cité dans Painchaud, op. cit., 168.

${ }_{12} \mathrm{Mgr} \mathrm{A}$. Taché, Esquisse sur le Nord-Ouest de l'Amérique (Beauchemin, Montréal, 1901), 175-81. On trouve en appendice la réponse de Mgr Taché, du 22 janvier 1894, à une série d'articles qui avaient paru dans Le Canada. 
Il faut le dire, pour justifier et disculper un peu nos compatriotes, on était loin de les encourager à venir ici. Mgr Taché avait été d'abord sous l'impression que les prairies de l'Ouest ne seraient jamais cultivables. Il avait exprimé cette pensée dans des lettres que nos journaux avaient reproduites et qu'ils publiaient encore quand ce grand évêque avait reconnu son erreur. Ces lettres ont grandement contribué à éloigner nos gens de l'Ouest canadien. ${ }^{13}$

Pendant les années 1880 , un certain nombre de prêtres et de laïques éminents hésitèrent de plus en plus à approuver l'émigration des Canadiens français du Québec dans les prairies. L'effondrement de la propriété immobilière, qui avait connu un boom au Manitoba, suivi du retour au Québec, en 1882 et 1883, de nombreux Canadiens français qui n'avaient pas trouvé "their pot of gold at the end of the rainbow", vint confirmer les doutes fortement enracinés qu'entretenaient plusieurs éléments de la société québécoise sur le caractère hospitalier des terres de l'Ouest ${ }^{14}$. "La persécution lente mais cruelle par laquelle on a voulu faire mourir, comme à petit feu, notre nationalité française (au Nord-Ouest)"15, la rapidité avec laquelle le gouvernement d'Ottawa avait écrasé la Rébellion du Nord-Ouest et l'exécution de Louis Riel dans l'atmosphère exacerbée d'une guerre raciale, tout cela contribua à convaincre les francophones du Québec que les Anglais, avec l'aide du gouvernement Macdonald, avaient atteint leur but: la conquête de l'Ouest.

Avant la politique de Sir John A. Macdonald, le Nord-Ouest était français. Aujourd'hui, tout notre or, qui eut pu être consacré à coloniser la province de Québec, a passé dans le NordOuest, dont on a fait à nos frais une terre anglaise. ${ }^{16}$

La création par le curé Labelle, en 1879, de la "Société de colonisation" destinée à promouvoir l'établissement de colons au nord de Montréal et l'apparition, cinq ans plus tard, sous le patronage de Mgr Duhamel, d'une semblable société dans le diocèse

13 Archives of Saskatchewan, Journal de Mgr O.-E. Mathieu, vol. 1 (1913). (Les Archives de l'Université Laval possèdent l'original de ce Journal.)

14 R. Painchaud, op. cit., 177. Mars.

15 Le Monde, 23 novembre 1885. Discours de F.-X.-A. Trudel sur le Champs de

16 Adolphe Ouimet, La vérité sur la question métisse au Nord-Ouest (Montréal, 1889), 397. Pour plus de détails, voir A. I. Silver, "The Metis Question", dans The West and the Nation by Carl Berger and Ramsay Cook (McLelland and Stewart, Toronto, 1916), 91113. 
d'Ottawa furent un coup direct porté aux grandioses projets de $\mathrm{Mgr}$ Taché relatifs à l'implantation d'une population francophone au Manitoba. On allait favoriser l'occupation du nord du Québec, puis la prise de possession graduelle de l'est et du nord de l'Ontario, de préférence à une colonisation canadienne-française dans les prairies. Répondant à un appel du curé de Saint-Jean-Baptiste, au Manitoba, qui demandait des colons, le Roi du Nord avait affirmé, en 1883: "Soyez tranquille, je m'en vais au Nord-Ouest avec mes gens des cantons de la 'Rouge'. Les deux rivières qui portent le même nom de la 'Rouge', la mienne et la vôtre, sont appelées à se joindre. Nous nous acheminons tranquillement vers les belles et fertiles régions de la baie James. Une fois là, nous nous donnerons la main."17

Le curé Labelle ne décourageait pas les Canadiens français de s'établir dans les prairies. Il recommandait vivement à ceux qui redoutaient les difficultés du défrichage des terres boisées de tenter fortune au Manitoba plutôt que de s'expatrier aux États-Unis. En 1885, il voyagea en Europe, sous les auspices du gouvernement fédéral et avec la bénédiction de Mgr Taché, pour faire connaître les avantages qu'offrait l'Ouest canadien à d'éventuels colons français, belges et suisses. Il accompagna même une petite délégation de citoyens français à Saint-Boniface à l'automne de 1885 . Toutefois, la majorité des gens de quelques importance avec qui il entra en contact en France prirent des terres dans le nord du Québec, et non point au Manitoba ${ }^{18}$. En outre, on peut croire qu'une lettre que lui écrivait en 1886 Onésime Reclus exprimait une façon de voir bien proche de la sienne: "Je suis convaincu qu'il faut surtout s'emparer d'Ontario, de façon à ce que le Canada français, condamné peutêtre à perdre le Nord-Ouest, soit au moins sûr d'aller de TerreNeuve à Saint-Boniface."19 Une ligne continue de colons francophones, qui s'allongerait du nord du Québec au Manitoba en passant par l'est et le nord de l'Ontario, serait plus utile à la survie culturelle des Français dans les prairies que le fait d'y installer un petit nombre de colons du Québec ou de la France. Pour le curé Labelle, l'achèvement du chemin de fer du Pacifique canadien ne faciliterait pas seulement le peuplement dans les prairies mais favoriserait la mise en oeuvre de son grand projet.

1930), 182. Roland Lamontagne, "Le Roi du Nord et sa suite française à Winnipeg",
CHAR (1954): 421 . février 1886; cité dans Gabriel Dussault, “Un réseau utopique franco-québécois et son projet de reconquête du Canada 1860-1891", Les Cahiers du Centre culturel canadien, \#3: 64. 
L'achèvement du chemin de fer du Pacifique canadien, la Rébellion de Riel et la diffusion, par les livres et les journaux, d'un nouvel ordre de priorité pour la colonisation francophone au Canada rendirent plus difficile le recrutement de colons canadiensfrançais du Québec par le clergé de l'Ouest. Quand, en 1888, l'abbé C.-A. Beaudry, du diocèse de Saint-Hyacinthe, demanda la permission d'entreprendre une tournée de conférences dans le diocèse de Montréal, aux fins de promouvoir la colonisation dans les prairies, l'archevêque, Mgr Fabre, la lui refusa ${ }^{20}$. En 1886, Jules-Paul Tardivel commença à publier dans son journal, La Vérité, une série d'articles dans lesquels il condamnait les efforts du clergé de l'Ouest pour attirer les Québécois au Manitoba. Tardivel s'éleva si bruyamment contre l'établissement de francophones dans l'Ouest canadien que Mgr Taché se crut obligé de lui écrire personnellement:

Vous paraissez avoir des haines à servir et je constate avec un amer regret que la passion vous aveugle... Votre journal a eu dans un temps mon admiration et mes plus vives sympathies mais pour être sincère je dois vous avouer que depuis quelques mois vous n'êtes certainement pas dans le vrai par rapport aux affaires du Nord-Ouest. ${ }^{21}$

Philémon Wright, G.-A. Nantel et plusieurs autres, dans des brochures et des articles de journaux, pressèrent leurs compatriotes d'occuper le nord du Québec et, par la suite, de prendre graduellement possession du nord de l'Ontario. Cela aiderait à empêcher "le départ d'un grand nombre de Canadiens pour le Nord-Ouest et les États-Unis"22.

Plusieurs dirigeants du Québec considéraient encore le Manitoba comme la seule solution viable qui s'offrît à l'habitant forcé de quitter sa province natale ou décidé à le faire. L'abbé C.-A. Beaudry, vicaire à la Présentation, dans le diocèse de SaintHyacinthe, visita le Manitoba avec un groupe de prêtres en 1887 et résolut, à son retour, de consacrer ses énergies et à l'établissement de francophones dans les prairies. Avec la bénédiction de son évêque, Mgr L.-Z. Moreau, et avec l'assentiment de Mgr Gravel et de Mgr Laflèche, l'abbé Beaudry fit le tour des diocèses de

20 R. Painchaud, op. cit., 188.

21 Archives de l'Archidiocèse de Saint-Boniface, Fonds Taché, A. Taché à J. P. Tardivel, Saint-Boniface, 27 avril 1856.

22 G.-A. Nantel, Notre Nord-Ouest provincial (Montréal, 1886), 34. 
Saint-Hyacinthe, de Nicolet et de Trois-Rivières, donnant des conférences sur la qualité du sol dans les prairies et sur les avantages que, dans les domaines de la culture et de la religion, le Manitoba avait à offrir à tous les colons qui songeaient à émigrer.

L'abbé Beaudry visita des régions du Québec en exhibant une lettre dans laquelle Mgr Laflèche approuvait non sans emphase ses efforts pour mettre un frein à l'exode des Canadiens français vers les États-Unis:

Quant à ceux que de pénibles circonstances forcent à laisser la province de Québec, c'est vers le Manitoba qu'ils doivent se diriger; là ils trouveront des terres toutes défrichées, de la plus grande fertilité et à des prix raisonnables, et surtout ils trouveront toutes les institutions du Canada pour l'éducation de leurs enfants et l'accomplissement de leurs devoirs religieux.

Je n'ai donc point d'objection à ce que vous l'abbé Beaudry veniez dans mon diocèse faire connaître à ceux qui le désireront les avantages qu'offre à la colonisation notre province de Manitoba, en les détournant en même temps de l'émigration aux États-Unis.

Je vous souhaite plein succès dans cette oeuvre qui sera certainement fort utile à nos compatriotes. ${ }^{23}$

Cette arme à la main, l'abbé Beaudry réussit à recruter environ 1,000 Canadiens français pour la province du Manitoba, en 1888 et au printemps de 188924 .

En 1890, l'élite de la seule province majoritairement francophone du Canada était unanime sur un point: les Québécois devaient rester au Québec; ceux qui étaient décidés à quitter la province devaient demeurer au Canada plutôt que d'émigrer aux États-Unis. Là où l'on différait d'avis, c'était sur l'endroit où ces émigrants devaient se fixer au Canada. Certains recommandaient le nord de l'Ontario, alors que d'autres soutenaient que le Manitoba offrait de meilleures perspectives. D'autres encore, comme J.-P. Tardivel, se plaignaient que des descriptions exagérément attrayantes des prairies, que l'on diffusait dans toute la belle province, eussent pour résultat le départ de beaucoup d'habitants qui auraient $\mathrm{pu}$, autrement, choisir de rester dans la province.

23 Le Manitoba, 7 mars 1889, l'abbé Beaudry au rédacteur.

24 Archives publiques du Canada, Agriculture Papers, 69243, A. A. Larivière to J. Carling, May 1, 1889. 
La suppression des écoles séparées, l'abolition du bilinguisme et les échecs juridiques et politiques qu'essuyèrent par la suite les Franco-Manitobains firent croître au Québec une ferme opposition à toute forme de propagande qui eût pu inciter les habitants des rives du Saint-Laurent à émigrer dans l'Ouest canadien.

J.-P. Tardivel reprit ses attaques contre les missionnairescolonisateurs qui recrutaient des colons pour le Manitoba, en affirmant que la migration de ses compatriotes vers les prairies était encore plus désastreuse que l'expatriation aux États-Unis, "car les Canadiens qui s'établissent dans la Nouvelle-Angleterre nous restent unis géographiquement”25. Un voyage qu'il fit au Manitoba en 1890 et l'hospitalité qu'affichèrent les Franco-Manitobains ne modifièrent pas ses idées. Il renouvela avec une vigueur accrue ses condamnations des agents qui oeuvraient au Québec en faveur de la colonisation dans l'Ouest. La belle province avait besoin de tous ses citoyens pour maintenir ses positions à la Chambre des Communes. En réduisant le nombre des votes au Québec et en augmentant le nombre des votes au Canada anglais, l'émigration des Canadiens français affaiblissait sérieusement l'influence des soixante-cinq députés du Québec à Ottawa. Les résidents francophones de l'Ouest étaient trop coupés du Québec et trop peu nombreux pour se défendre efficacement contre l'oppression. Le Québec ne pouvait pas se permettre d'envoyer d'autres victimes dans les prairies:

Il est même facile de prévoir que dans quelques années la persécution maçonnique deviendra tellement odieuse, tellement insupportable dans l'ouest, qu'il faudra ramener dans la province de Québec les groupes français de cette région. Et l'on veut grossir le nombre de ces infortunés qui deviennent de plus en plus de véritables exilés. ${ }^{26}$

De plus en plus de membres du clergé, à tous les degrés de la hiérarchie, se prononçaient contre l'activité des agents recruteurs de l'Ouest au Québec. L'archevêque Louis-Nazaire Bégin, par exemple, repoussa la demande de l'abbé Beaudry, désireux de faire une tournée dans le diocèse de Québec, en 1893. Il soutint que les Canadiens français de la belle province n'étaient pas attirés par le Manitoba. Les Québécois étaient plus intéressés à tenter fortune

La Vérité, 18 janvier 1890.

La Vérité, 13 juin 1896. 
aux États-Unis, tout simplement pour y accumuler le capital nécessaire à l'achat, dans leur province natale, d'une terre sur laquelle ils pourraient vivre confortablement ${ }^{27}$.

Les laïques en vue se mirent aussi de la partie. Le directeur général de la Société générale de Colonisation et de Rapatriement de Montréal, le docteur T.-A. Brisson, écrivit au premier ministre Wilfrid Laurier pour lui demander qu'un ordre fût donné aux missionnaires-colonisateurs de l'Ouest de restreindre leur activité au Québec:

He (l'abbé Osias Corbeil) is a large lymphatic, always tired of doing nothing and not possessing any aptitude as a recruiting agent. His role of emptying the Province of Quebec for the benefit of the West is at the same time seen unfavourably by the members of the clergy, who do not hesitate to close the doors of the presbyteries to him.

Abbé J. B. Morin carries on the same unpopular work as the Abbé Corbeil... I suggest respectfully that instead of allowing him to depopulate the Province of Quebec, he should be sent in the capacity of Agent of repatriation to the United States of the West...28

L'attaque lancée contre ceux qui participaient à la propagande visant à l'émigration d'habitants du Québec dans l'Ouest prit une telle ampleur que l'abbé Beaudry sentit le besoin de réfuter les accusations de Tardivel, en affirmant qu'il n'avait jamais dépeint les prairies comme une terre où coulaient "le lait et le miel" et qu'il n'avait jamais cherché à inciter les habitants qui étaient confortablement installés au Québec à quitter leur province natale 29 . L'abbé Beaudry fut puissamment secondé par l'abbé Georges Dugas, qui avait passé plus de vingt ans dans les prairies avant de revenir au Québec en 1889. Faisant le tour des paroisses dans le district avoisinant Montréal, il allégua qu'il était téméraire de recommander à d'honnêtes habitants, voués à leur travail, de s'établir sur des terres de roches dans des régions comme celle de Saint-Agathe. En dépit de leurs efforts, ces gens, incapables de joindre les deux bouts,

27 R. Painchaud, op. cit., 189.

28 A.P.C., Immigration Papers, No. 11680, Part I, Dr. T. A. Brisson to Wilfrid Laurier, August $27,1896$.

29 La Vérité, 18 janvier 1890. 
tombaient dans le découragement, abandonnaient leurs terres et cherchaient refuge dans les manufactures de la Nouvelle-Angleterre pour faire vivre leurs familles. Se fussent-ils établis au Manitoba d'abord, ils seraient restés sur le sol canadien ${ }^{30}$.

Le Monde rapportait en 1890 que les missionnaires-colonisateurs de l'Ouest faisaient leur travail de promotion au Québec avec la bénédiction de l'épiscopat tout entier, mais il est évident que tel n'était pas le cas ${ }^{31}$. Les seuls membres du haut-clergé québécois qui, pendant la crise des Écoles du Manitoba, restèrent fidèles à la lettre circulaire des évêques, de 1871, furent Mgr Moreau, de SaintHyacinthe, Mgr Gravel, de Nicolet, Mgr Blais, de Rimouski, et Mgr Laflèche, de Trois-Rivières ${ }^{32}$. Mis en présence d'un journaliste de La Minerve, après un voyage qu'il fit au Manitoba en 1892, Mgr Laflèche affirma que ni lui ni aucun de ses collègues n'encourageaient leurs compatriotes à quitter le Québec. Il insista sur le fait qu'il pressait simplement les Québécois décidés à aller gagner leur vie en dehors de leur province natale de s'installer dans l'Ouest canadien plutôt que de traverser la frontière pour tenter leur chance aux États-Unis ${ }^{33}$. C'est ainsi que $\mathrm{Mgr}$ Laflèche répondit à un appel à l'aide de Mgr Pascal, de Prince-Albert, en 1897, en lui prêtant les services d'un de ses prêtres, l'abbé J.-H. Brousseau, de Sainte-Annede-la-Pérade, comme missionnaire-colonisateur du district de la Saskatchewan ${ }^{34}$.

30 Le Manitoba, 16 avril 1890 et 3 septembre 1890.

31 Le Monde, 13 septembre 1890.

32 Avec l'assentiment de son évêque, Mgr Moreau, l'abbé Beaudry servit comme missionnaire-colonisateur pour le Manitoba jusqu'en 1893, année où il devint le procureur de l'évêque de Saint-Hyacinthe. Mgr Gravel visita le Manitoba à l'automne de 1890 et rapporta dans Le Monde (20 septembre 1890), par la plume de l'abbé F.-L. Desaulniers, qui l'avait accompagné pendant son voyage, qu'il était beaucoup plus facile de faire fortune au Manitoba que dans n'importe laquelle des paroisses les plus prospères du Québec. Mgr Blais répondit à une demande d'aide de la part des Manitobains, en 1899, en affirmant qu'il continuerait, comme il l'avait toujours fait, de donner aux membres de son clergé la directive de favoriser la migration de Canadiens français vers le Manitoba. Sa lettre est citée dans Painchaud, op. cit., 198-99.

33 La Minerve, 12 juillet 1892; cité dans R. Rumilly, Monseigneur Laflèche et son temps (Éditions B. D. Simpson, 1945), 349. Voir aussi Le Manitoba, 20 juillet 1892; La Vérité, 20 mai 1899. 1897.

34 A.P.C., Laurier Papers, No. 17201-02, Mgr Pascal à Wilfrid Laurier, 16 octobre 
Tant que la question des Écoles du Manitoba resta devant les tribunaux et qu'elle fut débattue dans l'arène politique, toute possibilité et tout espoir n'étaient pas perdus de voir restaurés les droits scolaires et linguistiques des Franco-Manitobains. La bien peu satisfaisante solution de compromis apportée à la crise des Ecoles du Manitoba par l'accord Laurier-Greenway et l'intervention du pape Léon XIII visant à faire accepter cet accord forcèrent même les partisans silencieux de la colonisation française dans l'Ouest à réexaminer leur position. En outre, la mort de $\mathrm{Mgr}$ Laflèche, en 1898, priva Mgr Langevin et ses cohortes du plus éminent et du plus éloquent avocat, au Québec, de la colonisation francophone dans les prairies.

Au même moment, l'épiscopat de l'Ouest décida d'intensifier son activité au Québec pour compenser la chute marquée du nombre des Européens de langue française qui allaient s'établir dans les prairies ${ }^{35}$. Cette décision n'eût pas pu survenir à un plus mauvais moment.

$\mathrm{Au}$ cours d'une des sessions du Congrès de colonisation organisé par la Société générale de Colonisation et de Rapatriement de Montréal, en 1898, on donna lecture d'une lettre de $\mathrm{Mgr}$ Langevin au docteur T.-A. Brisson. Elle contenait un vibrant appel au secours. Cet appel fut repris avec force par les délégués de l'Ouest canadien, le Père Zacharie Lacasse et l'abbé Moïse Blais, ce dernier missionnaire-colonisateur du diocèse du Manitoba, mais il ne suscita pas beaucoup de sympathie ${ }^{36}$. La vision du nord de l'Ontario, largement peuplé de Canadiens français, telle qu'évoquée par Mgr O'Connor, évêque de Peterborough, et par l'abbé Gingras, curé de Sturgeon Falls, éveilla plus d'intérêt parmi les délégués. Elle correspondait aux objectifs définis par le regretté Mgr Labelle, et plusieurs des principaux orateurs les reprirent dans leurs discours. Aucune des résolutions adoptées pendant le congrès n'avait trait à l'Ouest canadien; l'une portait sur la colonisation dans le district ontarien de Nipissing ${ }^{37}$. Bien qu'on ne puisse pas facilement en faire la preuve, il semblerait que plusieurs des délégués eurent le sentiment qu'il fallait à l'avenir préférer le nord de l'Ontario au Manitoba comme territoire de colonisation pour les Canadiens français décidés à quitter le Québec.

35 R. Painchaud, op. cit., 196.

36 Rapport du Congrès de la colonisation tenu à Montréal les 22, 23 et 24 novembre 1898 (La Patrie, Montréal, 1900), 54-58.

37 Ibid., 62-69. 
Membre du comité des résolutions pendant le congrès de colonisation de 1898, Tardivel continua par la suite de condamner, d'une plume trempée dans le vitriol, les efforts des missionnairescolonisateurs de l'Ouest pour recruter des colons au Québec. Mais il était maintenant disposé à accepter et même à approuver, avec quelques menues réserves, l'idée de la colonisation francophone dans le nord de l'Ontario:

Nous pouvons affirmer avec connaissance de cause que le clergé en général et tous les patriotes éclairés voient d'un mauvais oeil cette propagande des agents de l'Ouest.

Il nous semble tout à fait logique d'encourager plutôt le travail de colonisation dans le nord d'Ontario, parce que ces territoires nous touchent immédiatement, qu'en fait l'on recule, par degrés, les limites réelles de la province de Québec, et que la population qu'on y dirige, n'ayant point de solution de continuité, acquiert une double force pour nous. ${ }^{38}$

L'intensification de la propagande des agents colonisateurs de l'Ouest, en 1898, combinée avec la perte des droits scolaires et linguistiques au Manitoba, incita plusieurs journalistes à suivre la voie de Tardivel. L'Événement notait que cette propagnande "se (faisait) sur une trop grande échelle" et qu'il y avait déjà trop de Canadiens français dans ces régions ${ }^{39}$. Le Pionnier, La Presse et beaucoup d'autres journaux dénoncèrent coup sur coup l'activité des agents de colonisation de l'Ouest. Le rédacteur du Progrès du Saguenay était particulièrement irrité. Il blâmait amèrement le zèle déployé par ces agents, qui visitaient non seulement les vieilles paroisses de la province, mais aussi les régions de colonisation éloignées. Ces prêtres ne réussissaient que trop souvent à convaincre de naifs habitants de laisser la sécurité du Québec pour les lointaines prairies, où ils seraient soumis à la tyrannie de la majorité anglaise. Une activité comme celle-là n'était vraiment pas patriotique ${ }^{40}$. D'autres journaux, tels Le Journal et Le Courrier de SaintHyacinthe, affirmaient qu'il y aurait toujours des habitants qui quitteraient le Québec et qu'ils devaient être encouragés à rester sur le sol canadien, en se fixant de préférence au nord de l'Ontario, mais que les prairies présentaient aussi beaucoup d'avantages ${ }^{41}$. Le seul

\footnotetext{
$38 \quad$ La Vérité, 15 avril 1899.

39 L'Événement, 30 mai 1899.

40 Le Progrès de Saguenay, 25 mai 1899; cité dans La Vérité, 3 juin 1899. 1901.

41 Le Courrier de Saint-Hyacinthe, 6 mars 1900; Le Journal, 9 janvier 1901 et 18 avril
} 
journal qui, après 1898, encouragea d'une façon continue la migration de Québécois vers l'Ouest canadien fut $L a$ Patrie d'Israël Tarte. Â cause de son attitude, il dut subir les diatribes des membres du clergé et des autres journaux ${ }^{42}$.

Parce qu'ils craignaient une perte de revenus ensuite du départ de leurs paroissiens et aussi pour des raisons d'ordre national, de plus en plus de membres du clergé s'opposèrent privément à la diffusion de la propagande sur l'Ouest ou la condamnèrent publiquement. Un prêtre indigné écrivait au rédacteur de La Vérité: "Vidons la province de Québec, la seule que redoutent les Anglais et allons nous noyer là-bas (dans les prairies). O bêtise!"43 Le Pionnier rapporte dans l'un de ses numéros que l'abbé Georges Dugas, qui s'était fait l'avocat de la colonisation francophone au Manitoba au début des années 1890 , en était venu, après 1896, à la conclusion que les colons, en quittant le Québec, devaient se fixer dans le NouvelOntario plutôt que de se disperser dans les vastes espaces des prairies ${ }^{44}$.

L'encouragement donné à la colonisation dans le nord de l'Ontario et la condamnation des efforts visant à augmenter la population francophone de l'Ouest canadien prirent des proportions d'épidémie, selon le rédacteur du Manitoba:

Chose étrange, par toute la province de Québec on peut librement et autant de fois qu'on le désire, donner des conférences, écrire sur les feuilles publiques, faire directement et indirectement mille et une réclames en faveur de l'Algoma, du Nipissing et personne ne trouve à se plaindre. Mais l'on ne peut pas parler de l'Ouest sans se faire critiquer. Pourquoi cet ostracisme?45

Quelques-uns de ceux qu'on critiquait ainsi signèrent des réfutations, dirigées principalement contre J.-P. Tardivel. Dans des lettres qu'ils lui adressèrent directement ou qui furent publiées dans divers journaux, - La Presse, La Minerve, La Patrie, Le Trifluvien et La Vérité, - l'abbé D. Gérin, curé de Saint-Justin, et le Père Zacharie Lacasse, vieil ami de Tardivel, soutinrent qu'il y aurait toujours des gens pour quitter le Québec et que, si eux-mêmes et

\footnotetext{
42 L'Événement, 17 mai 1899; La Patrie, 10 février 1899.

43 La Vérité, 13 mai 1899.

44 Le Pionnier, 21 avril 1899.

45 Le Manitoba, 31 mai 1899.
} 
d'autres comme eux ne les approchaient pas pour leur faire voir les avantages économiques et religieux qu'il y avait à s'établir dans les paroisses françaises des prairies, ces gens traverseraient la frontière pour se fixer aux États-Unis ${ }^{46}$.

L'archevêque et les évêques de l'Ouest canadien furent troublés par cette réaction négative du Québec à leurs efforts pour amener un plus grand nombre de Canadiens français à s'installer dans les prairies. À la suite d'un voyage en Europe, Mgr Pascal, de Prince-Albert, rendit visite à l'archevêque de Montréal, Mgr Bruchési, pour solliciter son appui à la colonisation canadiennefrançaise dans les prairies ${ }^{47}$. La réponse qu'il reçut ne dut pas être encourageante. Moins d'un mois plus tard, tous les évêques canadiens de l'Ouest se réunirent à Calgary et rédigèrent une lettre destinée à leurs collègues du Québec, les suppliant de seconder leurs efforts:

Laisserons-nous ces étrangers s'emparer de tout le sol que le Gouvernement du pays livre aux nouveaux colons, à des conditions si avantageuses? Plus tard serait trop tard. C'est le moment de faire un effort décisif. C'est le moment d'assurer à l'élément de Foi catholique et de langue française, sa part de prépondérance, dans ce pays...

C'est dans cette pensée, Messeigneurs, que nous nous adressons, encore une fois, à vos Grandeurs, pour vous demander le secours de votre influence dans le but de favoriser le développement de la colonisation du Nord-Ouest... Ce que nous demandons c'est que vous veuillez bien recommander à votre clergé de faire des efforts pour que ce courant d'émigration, au lieu de franchir la frontière du Canada, se dirige, au contraire, vers les vastes plaines de l'Ouest. ${ }^{48}$

Des requêtes de cette sorte, non plus que d'autres, provenant de diverses agences catholiques de l'Ouest, à cette époque, n'obtinrent beaucoup de réponses favorables. De tous les évêques et archevêques du Québec, seul Mgr Blais, de Rimouski, promit son

46 Le Trifluvien, 5 mai 1899; La Vérité, 22 avril 1899; La Presse, 25 mars 1899; La Minerve, 23 mars 1899; La Patrie, 13 avril 1899.

$47 \quad$ La Presse, 9 février 1899.

48 Archives of Alberta, Fonds des Oblats, Box 94, D IV-505, Lettre de l'Archevêque et des Evêques de la Province de St-Boniface aux Vénérables Archevêques et Êvêques des Diocèses de la Province de Québec (mars 1899). 
aide et celle de son clergé pour détourner vers l'Ouest canadien le flot des Canadiens français qui émigraient aux États-Unis. Plusieurs n'accusèrent même pas réception de ces appels au secours ${ }^{49}$.

$\mathrm{Au}$ tournant du siècle, les représentants de l'intelligentsia québécoise ne considéraient plus, en grande majorité, le Manitoba et les Territoires du Nord-Ouest comme des régions convenables aux Canadiens français. Il valait mieux s'installer dans l'Ouest que de s'expatrier, mais, du fait qu'il était à proximité du Québec, l'Ontario offrait une plus grande sécurité dans le domaine culturel. Avant tout, les Canadiens français devaient rester chez eux. Le Québec disposait d'assez de terre pour faire sa part à chacun. Si les paroisses francophones, dispersées dans les prairies, avaient besoin de nouvelles recrues pour renforcer leurs positions, les agents de l'Ouest devaient concentrer leurs efforts sur le rapatriement des Canadiens français des États-Unis au lieu d'essayer de vider la province de Québec.

Les missionnaires-colonisateurs de l'Ouest canadien affrontèrent une forte concurrence aux États-Unis. Avec l'aide financière des gouvernements fédéral et provincial, la Société générale de Colonisation et de Rapatriement de la Province de Québec y envoya des représentants, distribua feuillets et brochures, et versa des gratifications aux journalistes franco-américains pour les inciter à promouvoir le rapatriement, soit au Québec, soit au nord de l'Ontario ${ }^{50}$. La Société de colonisation du Lac Saint-Jean, fondée en 1897 , affirma avoir réussi à rapatrier 5,706 personnes, de 1898 à 190451. La Société d'immigration de la vallée de l'Outaouais et la Société de rapatriement de l'Ontario fusionnèrent en 1904, sous le nom de Société d'immigration d'Ottawa, pour coordonner le recrutement d'expatriés francophones destinés aux districts de Nipissing, de Sudbury, etc... ${ }^{52}$ En plus de la concurrence que leur

49 R. Painchaud, op. cit., 197-99.

50 A.P.C., Immigration Papers, No. 11680, Part I-VII. Ces documents portent sur l'activité de cette société de 1894 à 1917. La société approuva la création d'une nouvelle colonie dans la région voisine de Verner, Ontario, en avançant des fonds à l'abbé C.-A.-M Paradis, qui recrutait des colons dans le nord du Michigan. Voir A.P.C., Immigration Papers, vol. 107, File 18296.

s1 A.P.C., Immigration Papers, No. 28703, Rapports annuels de la Société de colonisation du Lac Saint-Jean, de 1898 à 1907. La Société soufflait probablement ses chiffres pour justifier les subventions qu'elle recevait du gouvernement fédéral. Même l'Événement du 8 mars 1900 mit en doute l'exactitude de ces statistiques. 25,1904 .

2 A.P.C., Immigration Papers, No. 304593, vol. I, R. Chevrier to C. Sifton, March 
faisaient d'autres régions dans le domaine du rapatriement, les agents de l'Ouest durent combattre les mythes relatifs au climat des prairies $^{53}$ et l'opposition des membres des professions libérales et du clergé des États-Unis, qui jugeaient les Français de l'Ouest canadien trop faibles numériquement et trop dépourvus de droits constitutionnels pour pouvoir survivre ${ }^{54}$. N'obtenant que peu de succès dans le domaine du rapatriement, les missionnaires-colonisateurs augmentèrent le nombre des recrues destinées aux prairies à même la province de Québec 55 .

De 1901 à 1913, l'élite du Québec se désintéressa plus ou moins du travail poursuivi dans la belle province par les agents de l'Ouest. Joseph Girard, le député fédéral de Chicoutimi et du Saguenay, laissa entendre aux autorités de l'immigration, à Ottawa, que certains prêtres qui donnaient des conférences sur l'Ouest faisaient piètre impression au Québec ${ }^{56}$. C'est à peine si les membres de l'épiscopat mentionnèrent la colonisation pendant cette période ${ }^{57}$. À l'exception de La Patrie, les journaux consacraient rarement des éditoriaux à l'agriculture ou à la colonisation francophone à l'extérieur du Québec. La Presse et quelques autres quotidiens parmi les plus importants publièrent des annonces payées par des agents colonisateurs de l'Ouest. Plus que la colonisation, l'industrialisation était considérée pendant cette période comme le catalyseur qui provoquerait la prospérité économique, diminuerait l'expatriation et donnerait aux Canadiens français la possibilité de jouer un rôle important dans leur province et dans leur pays ${ }^{58}$.

53 A.P.C., Agriculture Papers, No. 2395, Rev. C.A. Beaudry to the Minister of Agriculture, September 1, 1899. Beaudry affirmait dans cette lettre que les Franco-américains sont "under the impression that the Province of Manitoba is a frightful country (a true Siberia)".

54 A.P.C., Immigration Papers, No. 22450, Rev. O. Corbeil to the Superintendant of Immigration, November 30, 1906. Le Manitoba du 28 mai 1902 cite un article de l'Indépendant de Fall River qui illustre l'hostilité des membres du clergé et des professions libérales au rapatriement qui se ferait au profit des prairies.

55 En 1901, 310 Canadiens français du Québec s'établirent au Manitoba et 249 furent rapatriés, selon La Patrie du 4 mars 1902, qui citait des statistiques publiées dans $L ' E ́ c h o d u$ Manitoba. La Société Saint-Jean-Baptiste du Manitoba fit une étude qui montra que, en 1900, 560 Canadiens du Québec s'étaient installés au Manitoba, alors qu'on comptait seulement 153 rapatriés. Voir R. Painchaud, op. cit., 199. $29,1908$.

${ }_{56}$ A.P.C., Immigration Papers, No.643583, Joseph Girard to W. D. Scott, August

57 W. F. Ryan, The Clergy and Economic Growth in Québec, 1896-1914 (P.U.L., Québec, 1966), 194-95.

58 Ibid., 194-95. 
Membres du clergé, journalistes et hommes politiques étaient bien trop préoccupés par la question de l'industrialisation et par les problèmes d'urbanisation qu'elle occasionnait pour accorder beaucoup d'attention à l'activité des missionnaires-colonisateurs de l'Ouest. Le nombre des habitants qui traversaient la frontière pour vivre aux États-Unis étant de beaucoup tombé, le départ annuel de quelques centaines de colons pour les prairies ne soulevait guère d'inquiétude.

À partir de 1913, le rythme de l'industrialisation se ralentit et les conditions économiques se détériorèrent lentement. La crise industrielle s'aggravant en 1914, l'agriculture et la colonisation devinrent une fois de plus les principaux moyens de réduire les départs pour les États-Unis, dont le nombre avait passé de 95,000 personnes pendant la première décennie du XXe siècle à 183,000 pendant la suivante ${ }^{59}$. Soudain, les missionnaires-colonisateurs de l'Ouest canadien, dont on s'était fortement désintéressé pendant la période de prospérité de la décennie précédente, devinrent, à partir de 1914, la cible de critiques et d'attaques verbales. Théo Hamel, l'agent de colonisation du gouvernement canadien à Québec, envoya à plusieurs reprises des lettres aux autorités de l'immigration, à Ottawa, pour exprimer son opposition à l'activité des agents colonisateurs de l'Ouest. Joignant à ses lettres des coupures de journaux pour étayer ses griefs, il affirmait qu'il était peu équitable de comparer le Québec au Nord-Ouest et que "the Province of Quebec principally has paid dear enough in men and money to help building (sic) the North West"60. Le ministre des Postes, LouisPhilippe Pelletier, se plaignit aussi qu'à cause de la sorte de propagande que quelques membres du clergé de l'Ouest diffusaienı par le moyen de la presse, beaucoup d'habitants qui auraient pu rester dans leur province natale s'en allaient dans les prairies ${ }^{61}$. Ensuite des plaintes de Pelletier, en particulier, le ministre de l'Intérieur, le docteur W. J. Roche, décida de déplacer le quartier général montréalais des missionnaires-colonisateurs vers un centre, encore indéterminé, des États-Unis.

59 Yolande Lavoie, L'Émigration des Canadiens aux États-Unis avant 1930 (P.U.M., Montréal, 1972), 39.

60 A.P.C., Immigration Papers, No. 535325-1. Theo Hamel to W. D. Scott, February 23, 1914, and March 13, 1914. $2,1914$.

${ }_{61}$ A.P.C., Immigration Papers, No. 535324-1, W. D. Scott to Dr. W. J. Roche, April 
Quand ils apprirent ce projet du docteur Roche, Mgr Langevin, Mgr Légal et Mgr Pascal s'y opposèrent vivement ${ }^{62}$. Les pressions exercées par l'épiscopat de l'Ouest et la promesse faite aux autorités, et en particulier au ministre L.-P. Pelletier, que les missionnaires-colonisateurs ne tenteraient décidément pas de dépeupler la province de Québec eurent pour résultat la mise au rancart du projet de déménager le quartier général des missionnaires-colonisateurs.

Les agents de colonisation qui représentaient des diocèses de l'extérieur du Québec résistèrent à la tempête de 1914, mais chaque fois que des annonces payées ou des lettres ouvertes signées par les abbés Giroux, Normandeau ou Bouillon étaient publiées dans la presse, se renouvelait le même scénario. En 1915, le docteur Eugène Paquet, député fédéral de l'Islet, écrivit au surintendant de l'Immigration d'Ottawa, W. D. Scott, pour blâmer l'activité de l'abbé Normandeau ${ }^{63}$. Il y eut tant de plaintes de cette nature qu'en 1917 le ministre de l'Intérieur, le docteur Roche, étudia avec les hauts fonctionnaires de son ministère la possibilité d'abolir le système des missionnaires-colonisateurs. Si on ne le fit point, on rappela sévèrement aux prêtres recruteurs qu'ils devaient d'abord faire porter leurs efforts sur le rapatriement.

Moins d'un an après la fin de la première guerre mondiale, l'Association catholique de la Jeunesse canadienne-française organisa un congrès pour créer "un grand mouvement d'intérêt et de sympathie en faveur de la colonisation" 64 . Les directeurs de l'A.C.J.C. décidèrent qu'il était temps de ranimer l'intérêt pour la colonisation, un secteur qui avait langui par suite de l'industrialisation et de la guerre.

Restons chez nous: tel fut le thème que développèrent la majorité des conférenciers, et, parmi eux, Honoré Mercier, le ministre de la Colonisation du Québec ${ }^{65}$. Après le Québec, le nord

62 Archives of Saskatchewan, A.C.F.C. Papers, vol. 56, Mgr. Langevin to Dr. W. J. Roche, June 2, 1914. A.P.C., Immigration Papers, no 535325-1, Mgr. Émile Légal, Bishop of Saint Albert, to Dr. W. J. Roche, June 6, 1914.

63 A.P.C., Immigration Papers, No 535325-1. Dr E. Paquet à W. D. Scott, 25 novembre 1915 .

${ }^{64}$ Le Problème de la colonisation au Canada français, Rapport officiel du Congrès de Colonisation tenu par l'A.C.J.C. à Chicoutimi, du 29 juin au 2 juillet 1919, Bureaux de l'A.C.J.C., (Montréal, 1920), ix.

ibid., 38-46. 
de l'Ontario était, pour un Canadien français, le meilleur endroit où s'installer. Cette prise de position correspondait parfaitement à l'ordre de priorité qu'avaientt établi à Longueuil les directeurs de l'A.C.J.C., au cours d'une réunion préliminaire convoquée pour préparer le congrès lui-même, qui fut tenu à Chicoutimi. Selon J.-E. Laforce, un agent du gouvernement canadien qui oeuvrait à Fall River, au Massachusetts,

it has been decided to push colonization in Northern Ontario, and every effort would be made to arrest colonization in Western Canada in view of the fact that it is not advantageous from a personal standpoint or from the nationalistic point of view to go west of the Great Lakes. ${ }^{66}$

Paul Boucher, un fermier de Boucherville, exprima probablement la pensée de la majorité des délégués en affirmant:

À mon humble avis, il vaut mieux prendre d'abord possession du nord de l'Ontario... Car, plus loin, les Canadiens français ne peuvent pas, malgré leur fécondité, devenir en nombre suffisant pour tenir tête aux immigrants étrangers...

On nous parle de souder l'élément acadien aux Canadiens français de l'extrême Ouest... C'est une perspective magnifique! Mais le vieux proverbe dit: "Qui trop embrasse, mal étreint." En justice pour nous-mêmes, nous n’avons pas le droit de risquer la partie, d'abandonner le rendement certain pour nous lancer, à tout hasard, dans la spéculation incertaine, sinon téméraire. Nous pourrions nous estimer satisfaits si la région de Nord-Ontario appartenait à la race canadienne-française. ${ }^{67}$

Le Règlement XVII n'était pas considéré comme un obstacle.

En 1919, l'intelligentsia du Québec avait clairement ordonné ses objectifs. L'habitant devait demeurer en terre québécoise. Il pouvait, si nécessaire, deménager dans l'une des villes de la province pour travailler dans une manufacture. S'il se sentait obligé de quitter le Québec, il devait s'établir dans le nord de l'Ontario. Il ne devait considérer l'émigration dans les prairies que comme une solution de dernier recours pour éviter l'expatriation. Cet ordre de priorité fut maintenu jusqu’à la crise économique des années 1930.

1919.

66 A.P.C., Immigration Papers, No. 535325-2, J.-E. Laforce to W. D. Scott, June 11,

67 Le Problème de la colonisation au Canada français, 92. 
Plus d'un prêtre, parmi les promoteurs de l'émigration francophone dans les prairies, se vit refuser l'accès à la chaire des églises ou l'hospitalité des presbytères pendant les années 1920. À l'un d'entre eux, qui s'était tourné vers lui pour solliciter son aide, Mgr Mathieu répondit:

Si vous avez sous la main les lettres pastorales des évêques de Québec, vous pourriez lire celle du mois d'octobre 1871 à propos de la colonisation du Nord-Ouest. Elle vous donnerait une bonne réponse à nos gens de Chicoutimi. 68

Une réponse comme celle-là montre que le clergé de l'Ouest ne comptait plus sur un afflux de Québécois pendant les années 1920. Les missionnaires-colonisateurs eux-mêmes reconnaissaient que les bons homesteads se faisaient rares et que, par ailleurs, ils devaient lutter, pour obtenir des colons, contre l'immense force d'attraction de "l'Abitibi québécois et ontarien" 69 . En outre, pendant les mêmes années, des associations nationalistes, comme l'Association catholique franco-canadienne, considéraient le regroupement dans des paroisses françaises des colons francophones dispersés à travers les prairies comme tout aussi important que le recrutement d'habitants du Québec.

En 1931, environ 177,000 personnes d'origine française vivaient dans les trois provinces des prairies ${ }^{70}$. Or, pendant chaque décennie, à l'exception d'une seule, de 1871 à 1931, les départs du Québec dépassèrent en nombre la population francophone totale des trois provinces de l'Ouest en cette année 1931. Pourquoi si peu de Canadiens français se dirigèrent-ils vers les prairies?

Plusieurs membres en vue du clergé de l'Ouest affirmèrent que le premier responsable de l'échec d'une colonisation francophone massive dans les prairies était J.-P. Tardivel. Le Père Lacombe, à ce qu'on rapporte, aurait dit à des journalistes et au ministre canadien des Postes, Rodolphe Lemieux, que le rédacteur de $L a$ Vérité avait à lui seul sapé les grandioses projets du clergé de l'Ouest relativement à l'établissement d'une population francophone ${ }^{71}$. L'abbé Georges

\footnotetext{
68 Archives of Alberta, Fonds des Oblats, Box 27 BII-530, Correspondance de Mgr Mathieu, O.-E. Mathieu au Père ?. 2 août 1919.

69 L'abbé G. Bilodeau, "La Colonisation et l'A.C.F.C.", dans Quinze ans de Vie française en Saskatchewan (A.C.F.C., 1927).

70 Canada, Census Report, I: 1188.

71 Canada, House of Commons Debates, April 9, 1907, 6203.
} 
Bilodeau, le missionnaire-colonisateur du diocèse de Prince-Albert, se faisait l'écho des sentiments du Père Lacombe dans un discours prononcé devant les membres de l'Association catholique francocanadienne, en 1927:

Depuis que Tardivel, l'homme dont les intentions ont toujours été des plus droites et dont le travail sur certains points a été si fructueux, depuis que Tardivel qui pendant assez longtemps a donné le mot d'ordre au point de vue national dans la province de Québec, depuis, dis-je, que Tardivel s'opposant aux desseins de Monseigneur Taché et de Sir Georges (sic) Étienne Cartier, s'est prononcé contre la colonisation de l'Ouest par les Canadiens-français, il est resté dans le clergé une défiance contre les missionnaires-colonisateurs qui se sont obstinés à voir dans la colonisation de l'Ouest par les Canadiens-français un bienfait tant pour la foi catholique que pour la race et le pays tout entier. ${ }^{72}$

Cette affirmation, entre autres de même nature, reflète la croyance ou la supposition que l'intelligentsia du Québec était responsable de l'échec de la colonisation francophone dans les prairies. Si tel avait été le cas, peu d'habitants eussent traversé la frontière américaine pendant les années 1870 , au moment où le haut clergé pressait ses ouailles désireuses de quitter le Québec de penser à se fixer au Manitoba. Quand, au tournant du siècle, une partie importante de l'élite québécoise recommanda l'Ontario comme étant plus avantageux que les prairies pour l'habitant qui quittait la belle province, il y avait déjà plus de 160,000 personnes d'origine française dans cette province. Il semblerait que l'intelligentsia québécoise ne fît qu'approuver la direction prise par la masse, qu'accepter un fait accompli.

On peut se demander si l'ordre de priorité fixé par l'élite québécoise influença sérieusement le mouvement migratoire des habitants qui laissaient le Québec, ou si ces derniers n'étaient pas guidés par des considérations plus immédiates et plus pratiques. Comme A. I. Silver l'a montré dans French Canada and the Prairie Frontier 1870-1890, les habitants du Québec croyaient les prairies inhabitables, et voués à l'échec ceux qui tentaient d'y cultiver le sol. En outre, ils avaient le sentiment que, dans ces lointaines prairies, le Canadien français n'était guère en sécurité. Cependant, si l'immobilité était un mode de vie pour les ruraux canadiens-français,

72 L'abbé Georges Bilodeau, op. cit., 124-25. 
comme l'affirme Silver, pourquoi tant d'entre eux émigrèrent-ils aux États-Unis ${ }^{73}$ ?

D'abord, le Canadien français qui était contraint de quitter le Québec pour des raisons économiques n'aurait pas émigré dans les prairies, où, pensait-il, il connaîtrait des problèmes similaires. En allant aux États-Unis, il pouvait trouver un emploi. Deuxièmement, s'il devait quitter le Québec, il possédait peu ou point d'argent et n'avait pas le moyen de déménager sa famille et ses biens dans les prairies, quand le billet de chemin de fer, pour le voyage de Montréal à Edmonton, coûtait $\$ 42.30$ en $1899^{74}$. Il pouvait s'installer en Nouvelle-Angleterre ou en Ontario pour une fraction du coût de son établissement dans l'Ouest. Troisièmement, l'établissement dans les prairies, à plus de mille milles de sa patrie, revêtait un aspect d'irrévocabilité, alors que le fait de vivre en Ontario ou dans l'un des États de la Nouvelle-Angleterre, à proximité de la frontière canadienne, donnait au Canadien français un certain sentiment de sécurité. Les prairies, c'était l'isolement total de sa patrie. Comme l'affirmait récemment un homme politique éminent, la distance entre Régina et Montréal est plus grande que celle qui sépare Paris et Moscou.

(Traduction: André Vachon, s.r.c.)

73 A. I. Silver, "French Canada and the Prairie Frontier 1870-1890," CHR, L, 1, (March 1969): 11-36.

${ }_{74}$ La Patrie, ler mars 1899, l’abbé J.-B. Morin au rédacteur. 